\title{
Case reports for embryo banking: for women who want their own
}

\author{
Amishi Vijay Vora ${ }^{1 *}$, Purnima Nadkarni ${ }^{1}$, Pooja Nadkarni Singh ${ }^{2}$, Vaibhav Nadkarni', \\ Aditi Nadkarni ${ }^{1}$
}

\begin{abstract}
${ }^{1}$ Department of ART, Nadkarni Training Academy and Test Tube Baby Hospitals, Killa Pardi and Surat, Gujarat, India ${ }^{2}$ Director and consultant, $21^{\text {st }}$ Century Group of Hospitals, Surat, Gujarat, India
\end{abstract}

Received: 08 May 2019

Revised: 29 May 2019

Accepted: 12 June 2019

\author{
*Correspondence: \\ Dr Amishi Vijay Vora, \\ E-mail: dramishi89@gmail.com
}

Copyright: () the author(s), publisher and licensee Medip Academy. This is an open-access article distributed under the terms of the Creative Commons Attribution Non-Commercial License, which permits unrestricted non-commercial use, distribution, and reproduction in any medium, provided the original work is properly cited.

\begin{abstract}
Out of the many challenges in management of female factor infertility, poor responders and low response to stimulation in aged and even younger women, seems to be a common problem. It is very difficult to offer one particular management strategy or treatment protocol for optimum outcome in this group of women of poor responders. In a low resource set up, IVF (In vitro Fertilization) specialist doctors usually face a challenge in treating women with poor/ low ovarian reserve as ovum / gamete donation is considered as a taboo in various sections of society even today. Hence women insist on having an offspring of "their own" and vehemently deny ovum / gamete donations. In this article we discuss 2 cases of poor ovarian reserve retrospectively, who underwent multiple cycles of controlled ovarian hyperstimulation for embryo banking and ultimately achieved pregnancy. Both patients achieved pregnancy with the method of embryo banking. Embryo banking should be considered and discussed. Various articles have discussed the advantages and disadvantages of embryo banking or even oocytes accumulation. The advantages of this technique is patients with poor/low ovarian reserve get a chance to be pregnant with their own oocytes and also have a chance for vitrification of residual embryos. Another advantage in such patients is that the embryos can undergo PGS (Preimplantation Genetic Screening) techniques in cases of suspected genetic disorders. The disadvantage in a low resource set up like India is the cost of the treatment. Nevertheless, embryo banking and accumulation of oocytes should be given as an option for treatment of poor/ low ovarian reserve and could be considered as a ray of hope for all future mothers hoping for a child of "their own".
\end{abstract}

Keywords: Embryo banking, Gamete donation, In-vitro fertilization, Oocyte accumulation, Ovum donation, own, Poor/low ovarian reserve, Preimplantation genetic screening, Ray of hope, Vitrification

\section{INTRODUCTION}

Out of the many challenges in management of female factor infertility, poor responders and low response to stimulation in aged and even younger women, seems to be a common problem. The incidence surprisingly varies between 9 to $24 \%$ according to many studies 1 . This wide range of incidence is due to the absence of an obvious reports in literature. But in most articles, it is defined as suboptimal response of stimulation protocols for 
recruitment of sufficient numbers of follicles which are associated with lower quality and number of retrieved oocytes, cycle cancellation and significantly lower pregnancy rate. ${ }^{1}$

Despite numerous predictive tests for diminished ovarian reserve including age, (Bologna Criteria and POSEIDON), the poor ovarian response is diagnosed only when subjected to ovarian stimulation. As there is no uniform definition of poor response, this makes the clinical trials incomparable till date. ${ }^{2}$ There are different strategies in the management of poor responder patients to obtain a maximum number of oocytes. The various trials till date suffer from methodological flaws and thus regarded as high risk for bias. This makes it very difficult to offer one particular management strategy or treatment protocol for optimum outcome in this group of women. ${ }^{2}$

The old question still remains which investigates the best strategy or protocol for management of poor responders. These protocols include high doses of gonadotropins against the standard dose for normal responders, short and ultrashort, mini and micro dose flare up regimen of GnRH agonists, and GnRH antagonists. In addition, further approaches have been applied to potentiate the effect of the above mentioned protocols like administration of oestradiol in luteal phase of cycle, administration of recombinant LH during gonadotropin stimulation (in small doses). ${ }^{3}$ Addition of Recombinant LH did not increase clinical pregnancy rate but significantly increased Live Birth Rate, administration of growth hormone $(\mathrm{GH})$, oral administration of dehydroepiandrosterone (DHEA) as an androgen, improvement of ovarian blood flow and vascularity through administration of aspirin, natural IVF cycle or minimal ovulation stimulation strategies for simple and inexpensive management. ${ }^{4,5}$ There is no conclusive evidence on using recombinant $\mathrm{FSH}(\mathrm{rFSH})$ instead of urinary FSH in poor responder patients. ${ }^{6-10}$ Luteal initiation of FSH might increase the number of recruited follicles by opening the recruitment window in the late luteal phase of the preceding cycle. ${ }^{11}$ In a prospective trial however ${ }^{12}$, the results of luteal initiation of FSH had higher cycle cancellation rates and lower pregnancy rates.

Now recently oocyte and embryo banking has emerged for individualization of treatment protocol pertaining to needs of the patient.

Accumulation of vitrified embryos followed by FET can be considered as new strategy to improve pregnancy rates in ovarian responders. Accumulating vitrified oocytes over several stimulation cycles and fertilizing them later can give higher Live Birth Rate per patient treated and potentially reduce the dropout rate in women with Poor Ovarian Reserve. In a country like India where old world traditions still prevail, women with infertility are looked down upon and considering a gamete or ovum donation becomes an issue of self-respect. In such scenarios embryo banking or oocyte accumulation brings a relieving option for distressed women ${ }^{13}$. The aim of this article is to present case reports of poor ovarian reserve and to discuss the approach and strategies to improve outcome in both cases and the role of "embryo banking" in both cases and their outcome. This is a retrospective study of cases attending the OPD of Nadkarni Hospital and Test Tube Baby Centre, Killa Pardi and Surat in Gujarat over a period of 6-8 months.

\section{CASE REPORT}

Retrospective study of 2 cases of poor ovarian reserve.

\section{Case 1}

Mrs. KYD 34 years married since 10 years with secondary infertility but no live issues (spontaneous abortion 2 years back) with normal BMI came with Complaints of dysmenorrhea since 3 months in early 2017 .On examination: The Vitals were stable and on Per Abdomen examination the abdomen was soft, and nontender. On P/S examination there were endometriotic patches on the Cervix. And on per vaginal examination the uterus acutely anteverted and bulky size. The first Ultrasonography showed Adenomyotic uterus with multiple myomas with right ovarian cyst and left ovarian endometrioma with no antral follicular count. Her serum Anti-Mullerian Hormone (AMH) levels were-0.78 ng/ml and CA -125 was 11.74. And Serum Prolactin was 17.25 $\mathrm{mIU} / \mathrm{l}$ and Serum TSH was $5.42 \mathrm{mIU} / \mathrm{l}$. Husband Semen Examination was normal. Diagnosis was severe adenomyoma with bilateral endometriosis with poor ovarian reserve and dysmenorrhea. ManagementInjection luprolide $3.75 \mathrm{mg}$ depot was given for reduction of pain and size of uterus and endometriosis. Subsequently in the next month a laparoscopic myomectomy and separation of intestinal adhesions was done with bilateral endometrioma resection.

In the next month, ultrasonic examination was repeated which showed Antral Follicular Count to be 2-3 on right side and 2 on the left side, so subsequently in the next month Injection luprolide was given and after 21 days ultralong protocol was started with Recombinant FSH and Urinary FSH a total of 2400U and 1200U respectively. Serum AMH levels seemed to improve (1.06), and during ovum pick up 5 oocytes were retrieved out of which 1 was Metaphase II (MII) . Serum estradiol after stimulation was $208 \mathrm{pg} / \mathrm{ml}$. One fertilized. Embryo transfer was done with 1 Grade 1 embryo but subsequent $\beta$ hcg was negative.

She was counselled for ovum donation, but patient insisted for her own eggs. So, Embryo banking option was given. In her next cycle, MOS (minimal ovarian stimulation) protocol started. Stimulation was given with Tablet Letrozole $2.5 \mathrm{mg}$ for 5 days and Injection Human Menopausal Gonadotropin (uFSH+uLH) 150 u for 9 doses and Antagonist added when lead follicle was 14 $\mathrm{mm}$, followed by trigger of 500 IU rhCG. Serum 
Estradiol level was 486 . On Ovum pick up, 2 Oocytes were retrieved, MII oocytes were 2 in number, 2 Grade 1 embryos formed which were were frozen on day 3 for banking.

In Her $3^{\text {rd }}$ cycle , antagonist protocol was followed by giving Injection recombinant FSH 300 + urinary Human Menopausal Gonadotropin (HMG) 150 for 10 days. Serum estradiol level was 608. Oocytes retrieved 4 and MII were 3 out of which 2 fertilized. Hence 2 grade 1 embryo were frozen $(2+2)$. she started developing pain of endometriosis and adenomyosis hence she was the given injection luprolide in the next month. Her serum AMH level seemed to improve $(1.03 \mathrm{ng} / \mathrm{ml})$.

In her fourth cycle again minimal ovulation stimulation protocol was in-corporated and tablet Clomiphene Citrate $50 \mathrm{Mg}$ was given for 5 days followed by Injection HMG 300U for 8 days with Antagonist, this time her serum Estradiol was $400 \mathrm{pg} / \mathrm{ml}$. Number of Oocytes retrieved was 1, out of which MII-1, and NO Embryo fertilized hence cycle was cancelled.

In the next month patient was found to have thin endometrium hence intrauterine Platelet Rich Plasma was instilled on day 7 when endometrial thickness was 7.5 $\mathrm{mm}$. After endometrial thickness was brought to $9.2 \mathrm{~mm}$, frozen embryo transfer of all 4 banked grade 1 embryos was done. And Consequently 1 st $\beta$ hcg was positive$590 \mathrm{Miu} / \mathrm{ml}$. Now patient is pregnant with single intrauterine pregnancy of 14 weeks.

To Summarize, the patient had severe adenomyosis with grade 3-4 endometriosis and husband semen analysis was normal. She had poor ovarian reserve and had 1 failed ICSI cycle where 1 grade 1 embryos was transferred hence offered embryo banking. She had subsequently undergone 3 cycles for oocyte retrieval and thus had 4 grade 1 embryos. After careful evaluation and preparing the endometrium, transfer of all 4 grade1 embryos resulted in pregnancy. Now she has single live intrauterine pregnancy of 14 weeks.

\section{Case 2}

30 years Mrs. KVU Married for 5 years with primary infertility came to Infertility OPD with weight $95 \mathrm{~kg}$ and BMI 40. Her ultrasonography showed left ovarian cyst and antral follicle count of 2 And right ovary showed antral follicular count of 5 to 6 with small anterior wall subserosal fibroid and poor endometrium. Her Serum Anti Mullerian Hormone was low $(0.86 \mathrm{ng} / \mathrm{ml})$ and her Serum Prolactin was 23.24 Miu/ml. Husbands Semen Examination was normal. She was advised weight reduction and endometrial studies for 4 months while she lost $12 \mathrm{kgs}$. She then underwent diagnostic hysteroscopy where endometrial broad septum was seen, and resection was done. She was then given tablet Estradiol valerate and Tablet Cabergolin and oral antioxidants.
In the subsequent month she underwent her 1st cycle with antagonist protocol with Injection recombinant $\mathrm{FSH}$ 300U and Injection HMG 150U for 8 DAYS With 4 days injection Growth Hormone For 4 Days and antagonist followed by recombinant Human Chorionic Gonadotropin trigger. Her endometrium was built up to $10.5 \mathrm{~mm}$. Her serum progesterone was $0.434 \mathrm{ng} / \mathrm{ml}$ and serum Estradiol Was $426 \mathrm{ng} / \mathrm{ml}$. Two oocytes were retrieved out of which only one Metaphase II (MII-1) was obtained and 1 Grade 1 embryo was made and transferred but subsequent $\beta$ hcg was negative.

Hence the couple was counselled for further treatment and given tablet DHEA and tablet sildenafil and tab arginine for endometrial quality built up and reduction of weight. She also was given intrauterine Platelet Rich Plasma. She was counselled for ovum donation, but patient insisted for her own eggs. Thus, the option of Embryo banking was given.

So, in the next month she was stimulated with Antagonist protocol and Injection Recombinant FSH 300U and injection HMG $150 \mathrm{U}$ and injection Growth Hormone was also given. Endometrium was $11.5 \mathrm{~mm}$. Serum Estradiol was $728 \mathrm{pg} / \mathrm{ml}$ and total oocytes retrieved were 6 out of which 3 were metaphase II and 3 fertilised and thus 3 grade 1 embryos. Hence 3 embryos were vitrified.

In her Third cycle, antagonist protocol was again offered with injection recombinant FSH 300u and injection HMG $150 \mathrm{U}$ for 12 days and injection Growth Hormone was given for 7 days followed by Antagonist for 3 days. This time her Serum Estradiol levels were 1100pg/ml. Dual trigger was then given and 11 oocytes retrieved, out of which metaphase II (MII) were 5 Out of which 4 were fertilized hence 4 embryos vitrified.

The subsequent month endometrial monitoring was done, and downregulation given, and on day 12 progesterone was $0.18 \mathrm{ng} / \mathrm{ml}$. 7 Grade 1 embryos were thawed out of which 5 recovered. 3 grade 1 embryos transferred on day 16 of cycle and $2 \mathrm{kept}$ for blastocyst out of which 1 progressed hence sequential transfer done with 1 grade 1A Blastocyst. Her subsequent 1 st $\beta$ hcg was thus positive. Now she has ongoing single live intrauterine pregnancy of 24 weeks.

To Summarize, the patient had primary Infertility with poor ovarian response and high BMI and husband semen analysis was normal. She also had 1 failed ICSI cycle where 1 grade 1 embryo was transferred hence offered embryo banking. She had subsequently undergone 3 cycles for oocyte retrieval and at the end of 3 cycles she had 7 grade1 embryos. After careful evaluation and preparing of endometrium, transfer of all 3 grade1 embryos and 1 grade 1AA sequential blastocyst resulted in pregnancy. Weight reduction also played a big role in the process. Now she has single live intrauterine pregnancy of 24 weeks. 


\section{DISCUSSION}

Poor ovarian reserve management is a challenge, as the limited follicular reserve of these patients results in a small cohort of oocytes. ${ }^{14} \mathrm{~A}$ variety of regimens have been employed to optimize the ovarian response of these women, with disappointing results. ${ }^{15}$ Moreover, the dropout rate due to the low probability of becoming pregnant is high, which naturally leads to disappointment and frustration. ${ }^{16}$ An ideal treatment would provide a similar number of embryos for transfer as that produced by women responding adequately to ovarian stimulation. Therefore, a strategy for obtaining a similar number of oocytes as in normal responders is needed.

According to this study, "Accumulation of oocytes: a new strategy for managing low-responder patients an RCT BY :A. Cobo, Nicolás Garrido Juana Crespo Remohí José Antonio Pellicer". ${ }^{14}$ A comparison was done in women with low ovarian reserve willing to undergo vitrification and accumulation of oocytes (LR: Low ovarian reserve patients)- ACCU-VIT (willing to undergo oocyte accumulation and vitrification) group with another group where women with low ovarian reserve wanted subsequent embryo transfers in every fresh cycle (LR) group. According to this study high drop-out rate in women with a poor prognosis was noted in a regular assisted reproduction cycle. It is worth mentioning that all these patients were encouraged to try an additional attempt with their own oocytes, even though they refused. The great majority of them switched to ovum donation. Additionally, concentrating efforts and decreasing the need for further treatments (as only one ICSI procedure is performed) encourage the patients to continue to 'build' a larger cohort in order to mimic the situation of normoresponder patients. There is evidence that failure to undergo embryo transfer is significantly related to the probability of discontinuing the treatment; thus, strategies which increase the success of IVF/embryo transfer are also of benefit in terms of anxiety levels. ${ }^{16}$ The oocyte survival rate after warming was slightly lower than that described for young normoresponders, but was high nonetheless. ${ }^{14}$ The embryo quality of these zygotes was similar to that of fresh oocytes, thus providing the rationale for a strategy of accumulation and selection of embryos for transfer regardless of their origin (fresh or cryopreserved cohorts). As a result, more embryos were obtained and transferred in the LR-Accu-Vit group. Only LBR (live birth rate) was considered as a clinical outcome. Live Birth Rate /patient was higher in the Low Reserve-Accu-Vit group, which confirms the efficiency of this method for managing LR patients. Indeed, this study was able to treat all LR patients who chose the option of oocyte accumulation, achieving a very low embryo-transfer cancellation. The positive effects of this strategy are even more evident when cumulative outcome is considered. Larger oocyte/embryo cohorts in LR patients allow the cumulated success rate after cryotransfer of surplus cryopreserved embryos to be increased. In this study, the cost per live births in the LR- fresh group was around 5\% lower compared with the LRAccu-Vit group (data not shown). It is worth mentioning that in the LR-fresh group the population which underwent more than two stimulations is significantly lower than in the vitrification group. In consequence, the analysis is made in a population which was subjected to mainly one stimulation cycle; hence, the cost per newborn seems to be lower. Hence cost is a factor to be considered. According to another study Perhaps the only oocyte banking advantage is reduction in the cost of fertilization and embryo transfer for multiple cycles, although the cost of oocyte freezing in successive cycles and ultimately, their thawing compensate this cost reduction. The other advantage of oocytes/embryos banking in poor responders is for preimplantation genetics screening (PGS) (Preimplantation Genetic Screening) cycles. ${ }^{1,17}$ It is confirmed that the increase of aneuploidy rate in oocytes and resulting embryos is correlated with aging in women. ${ }^{16}$ However, the application of PGS to improve pregnancy rate in late reproduction age and also younger couples is uncertain and questionable. The focus of IVF clinics on PGS has waxed and weaned during the last decade due to its technical insufficiency, but it has risen again following introduction of next generation sequencing (NGS) technology and trophectoderm biopsy of blastocyst at the beginning of current decade. ${ }^{17}$

What is new? Dual stimulation- it is a protocol based on presence of multiple waves of follicular recruitment occurring regularly during normal menstrual cycle ${ }^{17}$.The follicles in each wave are similar but of not the same diameter. ${ }^{18}$ Stimulation in follicular or luteal phase of the same menstrual cycle resulted in the formation of similar number of blastocysts in low ovarian reserve patients. These blastocysts contributed to the transferable blastocysts hence contributing to the number of transfers per menstrual cycle. ${ }^{19}$

Some researchers may debate the use of dual stimulation in a single cycle (DUO-STIM) protocols for pooling oocytes in a single cycle would be an effective method, but more research is needed for proving the significance.

Another advancement is by Kawamura et al, showed that fragmentation of ovaries promoted actin polymerization and disrupted ovarian Hippo signaling, leading to increased expression of downstream growth factors and promotion of follicular growth and mature oocytes. This technique together with Akt stimulator treatment and autografting the cortex of the ovary is known as Ovarian Fragmentation and in vitro activation. ${ }^{20}$ This technique is complex and involves tertiary handling hence not yet easily available in India.

\section{CONCLUSION}

In a country like India, women face a taboo of "not bearing a child of her own" and in some cases the husband is never tested nor blamed. In such a society, 
women face the fear of rejection and abandonment if diagnosed with poor ovarian reserve. Thus, techniques to manage low ovarian reserve would be a boon to such patients. Oocyte accumulation and also embryo banking is an effective method of management in low reserve patients. The advantages of this technique are patients with poor/low ovarian reserve get a chance to be pregnant with their own oocytes and also have a chance for vitrification of residual embryos.

Another advantage in such patients is that the embryos can undergo PGS techniques in cases of suspected genetic disorders. So instead of giving a direct option of ovum donation, an alternative can be given to the patient.

The disadvantage in a low resource set up like India is that the cost of the treatment is significantly more than that of the fresh multiple stimulation cycles as there is no thawing and banking involved.

In all various studies show that the use of embryo banking may significantly improve oocyte retrieval and also clinical live birth rates in women with poor ovarian reserve and insisting on having a child of their own.

More RCTS are hence needed to reduce the dilemma and to come up with more cost-effective techniques for embryo banking or oocyte accumulation to reduce the burden of infertility in poor/ low ovarian reserve patients.

\section{ACKNOWLEDGMENTS}

Authors would like to thank patients without whom such case report could not be written.

\section{Funding: None}

Conflict of interest: None declared

Ethical approval: Not required

\section{REFERENCES}

1. Sadeghi MR. Oocytes/Embryos Banking: a vague hope for poor responder women. J Reprod Infertil. 2018;19(3):123-4.

2. Papathanasiou A, Searle BJ, King NM, Bhattacharya S. Trends in 'poor responder'research: lessons learned from RCTs in assisted conception. Hum Reprod Update. 2016;22(3):306-19.

3. Drakakis P, Loutradis D, Kallianidis K, Liapi A, Milingos S, Makrigiannakis A, et al. Small doses of LH activity are needed early in ovarian stimulation for better quality oocytes in IVF-ET. Eur J Obstet Gynecol Reprod Biol. 2005;121(1):77-80.

4. Ferraretti AP, Gianaroli L, Magli MC, D'angelo A, Farfalli V, Montanaro N. Exogenous luteinizing hormone in controlled ovarian hyperstimulation for assisted reproduction techniques. Fertil Steril. 2004;82(6):1521-6.

5. Bosdou JK, Venetis CA, Kolibianakis EM, Toulis KA, Goulis DG, Zepiridis L, et al. The use of androgens or androgen-modulating agents in poor responders undergoing in vitro fertilization: a systematic review and meta-analysis. Hum Reprod Update. 2012;18(2):127-45.

6. Ng EH, Lau EY, Yeung WS, Ho PC. HMG is as good as recombinant human FSH in terms of oocyte and embryo quality: a prospective randomized trial. Hum Reprod. 2001;16(2):319-25.

7. Westergaard LG, Erb K, Laursen SB, Rex S, Rasmussen PE. Human menopausal gonadotropin versus recombinant follicle-stimulating hormone in normogonadotropic women down-regulated with a gonadotropin-releasing hormone agonist who were undergoing in vitro fertilization and intracytoplasmic sperm injection: a prospective randomized study. Fertil Steril. 2001;76(3):543-9.

8. Gordon UD, Harrison RF, Fawzy M, Hennelly B, Gordon AC. A randomized prospective assessorblind evaluation of luteinizing hormone dosage and in vitro fertilization outcome. Fertil Steril. 2001;75(2):324-31.

9. De Placido G, Mollo A, Alviggi C, Strina I, Varricchio MT, Ranieri A, et al. Rescue of IVF cycles by HMG in pituitary down-regulated normogonadotrophic young women characterized by a poor initial response to recombinant FSH. Hum Reprod. 2001;16(9):1875-9.

10. Eskandar M, Jaroudi K, Jambi A, Archibong EI, Coskun S, Sobande AA. Is recombinant folliclestimulating hormone more effective in IVF poor responders than human menopausal gonadotrophins?. Med Sci Monitor. 2004;10(1):PI69.

11. Gougeon A. Regulation of ovarian follicular development in primates: facts and hypotheses. Endocrine Rev. 1996;17(2):121-55.

12. Rombauts L, Suikkari AM, MacLachlan V, Trounson AO, Healy DL. Recruitment of follicles by recombinant human follicle-stimulating hormone commencing in the luteal phase of the ovarian cycle. Fertil Steril. 1998 Apr 1;69(4):665-9.

13. Kwon H, Choi DH, Kim EK, Kim EH, Lee SE. Accumulation of Vitrified Embryos Followed by Frozen Embryo Transfer in Poor Ovarian Responders According to Bologna Criteria. Gynecol Obstet (Sunnyvale). 2015;5(10):1000331.

14. Cobo A, Garrido N, Crespo J, José R, Pellicer A. Accumulation of oocytes: a new strategy for managing low-responder patients. Reprod Biomed Online. 2012;24(4):424-32.

15. Schoolcraft WB, Surrey ES, Minjarez DA, Stevens JM, Gardner DK. Management of poor responders: can outcomes be improved with a novel gonadotropin-releasing hormone antagonist/letrozole protocol?. Fertil Sterility. 2008;89(1):151-6.

16. Surrey ES, Schoolcraft WB. Evaluating strategies for improving ovarian response of the poor responder undergoing assisted reproductive techniques. Fertil Steril. 2000;73(4):667-76. 
17. Cedars MI. Assisted reproductive technology: moving forward or just moving?. Fertil Steril. 2016;105(3):588-9.

18. Pierson RA, Ginther OJ. Ultrasonographic appearance of the bovine uterus during the estrous cycle. J Am Vet Med Assoc. 1987;190(8):995-1001.

19. Ubaldi FM, Capalbo A, Vaiarelli A, Cimadomo D, Colamaria S, Alviggi $C$, et al. Follicular versus luteal phase ovarian stimulation during the same menstrual cycle (DuoStim) in a reduced ovarian reserve population results in a similar euploid blastocyst formation rate: new insight in ovarian reserve exploitation. Fertility and sterility. 2016;105(6):1488-95.
20. Kawamura K, Cheng Y, Suzuki N, Deguchi M, Sato Y, Takae S, et al. Hippo signaling disruption and Akt stimulation of ovarian follicles for infertility treatment. Proc Natl Acad Sci USA. 2013;110(43):17474-9.

Cite this article as: Vora AV, Nadkarni $\mathrm{P}$, Singh $\mathrm{P}$ N, Nadkarni V, Nadkarni A. Case reports for embryo banking - for women who want their own. Int $\mathbf{J}$ Reprod Contracept Obstet Gynecol 2019;8:2926-31. 